\title{
Enhancement of the Kapitza Conductance at 0.32 meV Phonon Energy
}

\author{
U. Heim, R. J. Schweizer, ${ }^{*}$ O. Koblinger, M. Welte, \\ and $W$. Eisenmenger \\ Physikalisches Institut der Universität Stuttgart, Stuttgart, Federal Republic of Germany
}

(Received July 9, 1982)

Using quasimonochromatic relaxation phonons emitted by superconducting tunneling junctions, the phonon transfer through real solid-liquid helium interfaces was tested. We found an enhancement of the energy transmission if the incident phonons exceed the threshold energy $\mathrm{E}_{o}=0.32 \mathrm{meV}$. This sharp threshold energy shifts to $0.42 \mathrm{meV}$ if the lighter isotope ${ }^{3} \mathrm{He}$ is used.

\section{INTRODUCTION}

The experimental fact that the phonon transmission of interfaces between solids and liquid $\mathrm{He}$ is greater than predicted by the acoustic mismatch theory has been unexplained since $1941 .{ }^{1,2}$ Nevertheless, in the past 10 years new techniques have made it possible to determine experimentally the reflection properties of such interfaces at definite phonon energies. Using superconducting tunneling junctions as generators of quasimonochromatic phonons and as quantum detectors with definite energy threshold, ${ }^{3,4}$ it has been shown that the elastic reflection of highenergy phonons $(0.5-3.6 \mathrm{meV})$ by real solid surfaces is strongly reduced if the surfaces are covered with two or more atomic layers of helium. ${ }^{5,6}$ In contrast, in situ cleaved solid-helium interfaces show an elastic phonon scattering in accordance with the acoustic mismatch theory. ${ }^{7}$ Using $0.1-\mathrm{meV}$ phonon pulses generated by stimulated Brillouin scattering, Schubert et $a l^{8}$ reported on saturation of the phonon losses at high intensities. Based on these facts, $\mathrm{Kinder}^{9}$ proposed a theoretical model which explains the anomalous phonon losses at helium-covered interfaces by localized surface excitations. On the other hand, concerning the question of the microscopic nature of these hypothetical defects, the evidence is still poor.

*Present address: Max-Planck-Institut für Festkörperforschung, Stuttgart, Federal Republic of Germany. 
Schubert et al. observed the anomalous losses at $0.1 \mathrm{meV}$ only if the interfaces were contaminated with a hydrocarbon film.

Sabisky and Anderson ${ }^{10}$ determined the phonon reflection of $\mathrm{SrF}_{2}-\mathrm{He}$ interfaces in the range of $0.1-1 \mathrm{meV}$, using a spin-phonon spectrometer. They found a reflection minimum around $0.3 \mathrm{meV}$ if the samples were exposed to pump oil vapors, whereas clean samples showed a rapid but structureless and monotonic reduction of the reflectivity between 0.2 and $0.6 \mathrm{meV}$.

In the experiments reported in this paper we studied the energy transmission rather than the elastic reflection of solid-liquid helium interfaces. Using superconducting tunneling junctions as phonon generators, we varied the energy of the incident phonons mainly in the range from 0.2 to $1 \mathrm{meV}$. If the generator junction is covered by a He film, the transmitted energy causes an enhanced evaporation. If, alternatively, the sample is immersed in liquid ${ }^{4} \mathrm{He}$ below the $\lambda$ transition, the energy is converted into second sound. Both the enhanced evaporation and the second sound may be detected by a second superconducting tunneling junction.

\section{EXPERIMENTAL TECHNIQUES AND RESULTS}

Two parallel superconducting tunneling diodes (generator and detector), evaporated on single-crystal substrates (silicon or sapphire), are mounted opposite to each other at a distance of $d \simeq 1 \mathrm{~mm}$. Most of the experiments have been carried out with two tin junctions; experiments with an aluminum generator yielded the same results. The size of the diodes is $1 \times 2.5 \mathrm{~mm}$. The sample chamber can be filled alternatively with liquid $\mathrm{He}$ or He gas of a controlled pressure. Cooling is provided by a liquid ${ }^{4} \mathrm{He}$ bath surrounding the chamber, and temperatures down to $1 \mathrm{~K}$ can be reached. Before cooling down, the sample chamber is carefully evacuated by a diffusion pump in order to avoid contamination of the diode surfaces by frozen air.

In our experiments we used three kinds of energy transfer from the generator to the detector:

(i) The sample chamber is filled with He gas of low pressure, typically $p \leq 10^{-4}$ Torr at $1 \mathrm{~K}$. Helium atoms may fly ballistically from the generator to the detector, i.e., the mean free path of the He atoms $\lambda>d$. In this regime a pulse-like excitation of the generator causes a broad signal at the detector, which reflects the time-of-flight distribution (and consequently the energy distribution) of the $\mathrm{He}$ atoms evaporated from the He film covering the generator (Fig. 1). ${ }^{11}$

(ii) In the opposite limit, if the mean free path $\lambda$ of the He atoms in the He gas is short compared with the generator-detector distance $d$, a 


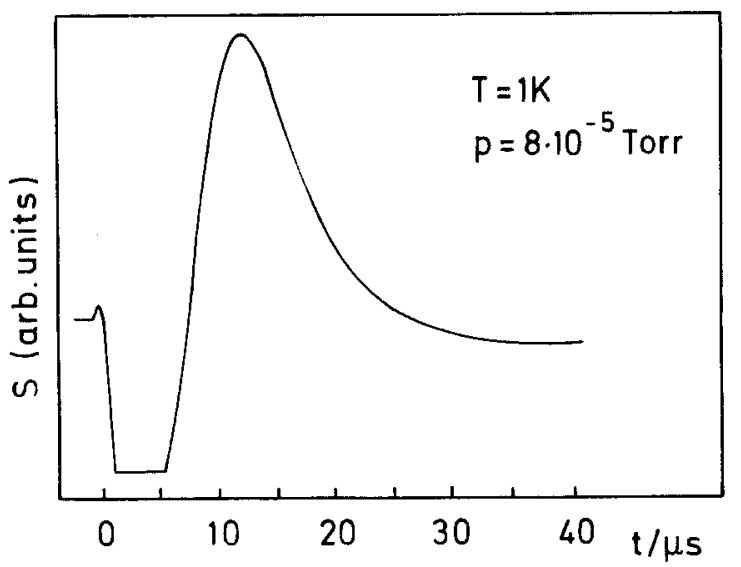

Fig. 1. Ballistic signal. Tin generator, Al detector, $d=1 \mathrm{~mm}$, generator pulse width $0.5 \mu \mathrm{sec}$.

pulse-like excitation of the generator produces a local pressure enhancement above the He film covering the generator. This pressure enhancement propagates as an adiabatic wave with the velocity of gas sound $v_{s}=$ $(\gamma k T / m)^{1 / 2}\left(\gamma=5 / 3\right.$ for $\mathrm{He} ; m$ is the mass of an He atom) (Fig. 2). ${ }^{11} \mathrm{~A}$ sequence of echoes is produced by reflections. Due to our geometry, a spherical wave rather than a plane wave is excited; consequently, a highpressure region is followed by a low-pressure region and the shape of the signal resembles the time derivative of the input pulse. ${ }^{11}$

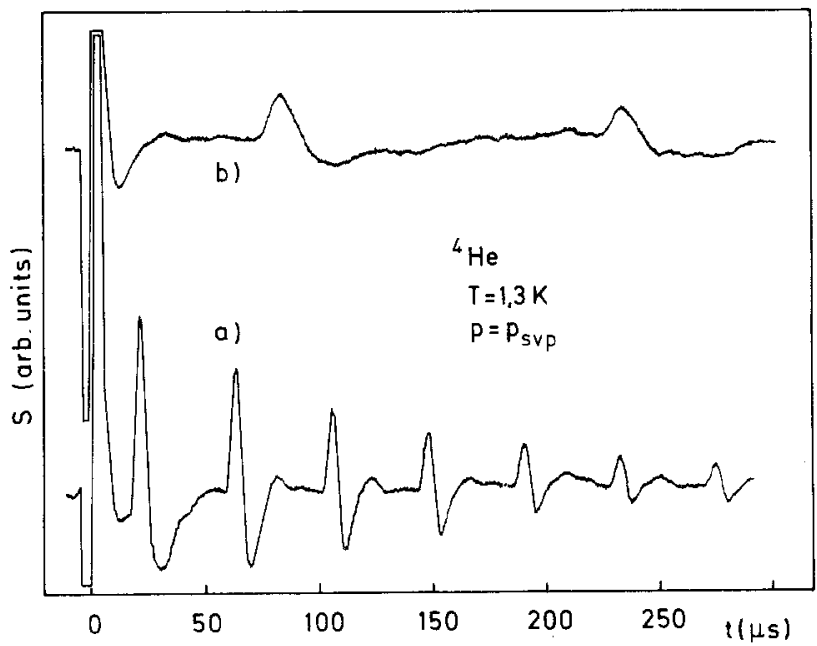

Fig. 2. (a) Adiabatic sound and (b) second sound. Tin generator and detector, $d=1 \mathrm{~mm}$, generator pulse width $4 \mu \mathrm{sec}$. 
(iii) If generator and detector are immersed in liquid ${ }^{4} \mathrm{He}$ below the $\lambda$ transition, the energy transfer is performed by a second-sound pulse. ${ }^{12}$ Also in this case a sequence of echoes arises and the shape of the input pulse is differentiated for the same reasons.

In all these experiments, the tunneling detector behaves like a fast bolometer recording short-time temperature variations, which in the cases (i) and (ii) are due to enhanced condensation during pulse arrival.

Considering the generator in these cases, there are two possibilities for the microscopic nature of the energy transfer in to the He gas: thermalization of the high-frequency phonons in the $\mathrm{He}$ film or in the solid-He interface and subsequent evaporation due to the enhanced film temperature, and, on the other hand, direct desorption of single He atoms by one-phonon processes. In the case of direct desorption, due to energy conservation, an onset energy is required, ${ }^{13}$ which for ${ }^{4} \mathrm{He}$ is $0.6 \mathrm{meV}+$ $k T \ln \left(p_{\text {svp }} / p\right)$, where $p_{\text {svp }}$ is the saturated vapor pressure. Our measurements in the second-sound range [case (iii)] were mainly motivated by the purpose of examining experimentally earlier attempts to interpret some of the structures observed in the ballistic and gas sound regimes by the assumption of direct desorption processes ${ }^{14}$ Further criteria of discernment are the characteristic pressure dependence of the desorption threshold energy and the occurrence of nonthermal time-of-flight distributions. ${ }^{15,16}$

In order to isolate the contribution of the quasimonochromatic relaxation phonons of energy $E=e V_{G}-2 \Delta_{G}$ ( $V_{G}$ is the generator bias voltage, $\Delta_{G}$ is the superconducting gap parameter of the generator), we determined the derivative of the signal with respect to the generator current $d S / d I_{G}{ }^{3,4}$ In the ballistic regime, where the generator is driven normal by dc currents, a puls: modulation technique must be used. Thereby the input pulse amplitude $A$ is slowly varied in the form $A(t)=A_{0}+\delta A \sin \omega_{M} t$; the signal is sampled by a boxcar integrator and finally analyzed by a lock-in amplifier. Typical values are: pulse width $0.5-3 \mu \mathrm{sec}$, repetition rate $\leq 1 \mathrm{kHz}, \nu_{M}=$ $\omega_{M} / 2 \pi \approx 150 \mathrm{~Hz}$. In the case of gas sound and second sound a better signal-to-noise ratio is achieved in biasing the generator with a dc current and a superimposed small modulation, i.e., $I_{G}=I_{0}+\delta I \sin \omega_{M} t$; thereby a sound wave of frequency $\nu_{M}$ is generated. Because of the differentiating behavior of our arrangement, it is advantageous to choose $\nu_{M}$ not too low, and the signal can further be enhanced by working near one of the acoustic resonance frequencies of the gas column between generator and detector, i.e., $\nu_{M} \simeq \nu_{n}=n v_{s} / 2 d$ (typically $\nu_{1} \simeq 30 \mathrm{kHz}$ ). In this case, the ac signal is directly rectified by a lock-in amplifier.

Typical results are given in Fig. 3. In the range of generator voltages $0<e V_{G}<2 \Delta_{G}+0.3 \mathrm{meV}$ the slope of $d S / d I_{G}$ can be fitted with the assumption that the signal $S$ is proportional to the electrical power $P=V_{G} I_{G}$ 


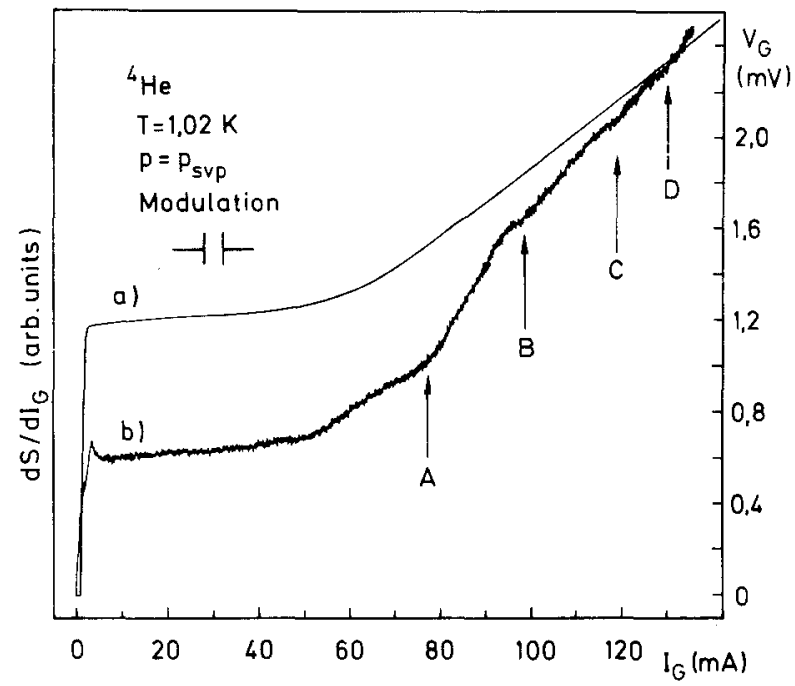

Fig. 3. Generator characteristic and (b) signal derivative in the adiabatic gas sound range. Tin generator and detector, $d=1 \mathrm{~mm}$, $\nu_{M}=25.7 \mathrm{kHz}$. The relaxation phonon energies are (A) 0.32 , (B) 0.65 , (C) 0.94 , (D) $1.11 \mathrm{meV}$.

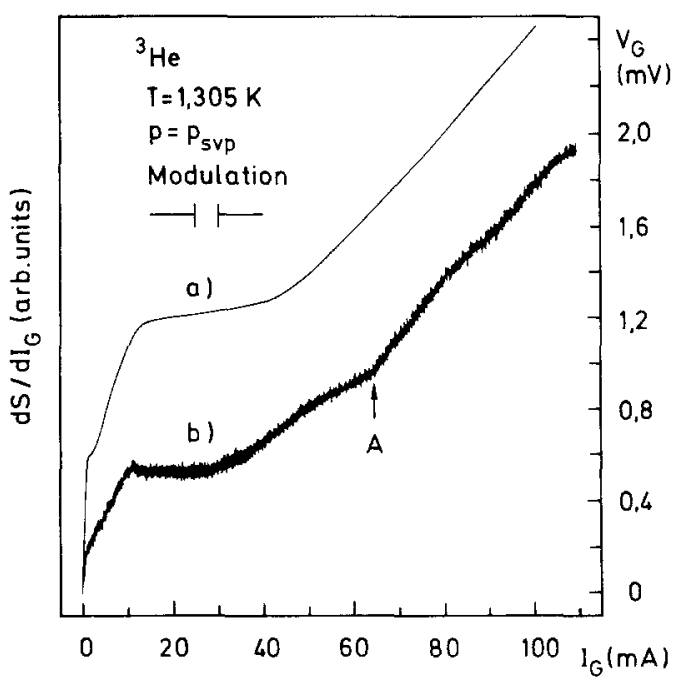

Fig. 4. (a) Generator characteristic and (b) signal derivative in the adiabatic gas sound range, using ${ }^{3} \mathrm{He}$ as transfering medium. The relaxation phonon energy at $\mathrm{A}$ is $0.42 \mathrm{meV}$. 
dissipated in the generator. When the relaxation phonon energy $E=$ $e V_{G}-2 \Delta_{G}$ reaches the threshold $E_{0}=0.32 \mathrm{meV}$ (Fig. 3, arrow A), a significant enhancement of the signal slope is observed. If the signal-to-noise ratio is optimum, further kinks are observed at $0.65,0.94$, and $1.11 \mathrm{meV}$ (Fig. 3, arrows B, C, D). The onset at $E_{0} \simeq 0.32 \mathrm{meV}$ is observed in the liquid $\mathrm{He}$, as well as in the $\mathrm{He}$ gas both in the ballistic and in the adiabatic wave regime. Using different generator junctions, we varied the power dissipated at $e V_{G}=2 \Delta_{G}+0.32 \mathrm{meV}$ by more than a factor of ten. Both tin and aluminum generators could be used as generator, and covering the generator with an evaporated $\mathrm{SiO}$ film $(3500 \AA)$ did not affect the onset at $0.32 \mathrm{meV}$. A significant shift of the threshold energy could be reached by changing the $\mathrm{He}$ isotope (Fig. 4, arrow A) and slighter shifts were observed if temperature and gas pressure were varied. The data are summarized in Table I.

A slope of $d S / d I_{G}$ consistent with the assumption $S \sim P=V_{G} I_{G}$ was obtained in two experiments: (i) using glass as the substrate for the junctions and (ii) contaminating the $\mathrm{He}$ gas filled into the sample chamber by a small amount of $N_{2}$. This can be explained by thermalizing phonon converting processes in the metal-glass interface or in the layer of frozen $N_{2}$ covering the generator. On the other hand, contaminating the $\mathrm{He}$ gas with $\mathrm{Ne}$ did not affect the observed anomaly. But if we filled the sample chamber before cooling down with $\sim 1$ liter $\mathrm{H}_{2} \mathrm{STP}$, the onset energy shifted to $0.25 \mathrm{meV}$. These preliminary measurements will be continued.

TABLE I

Summary of the Observed Threshold Energies ${ }^{a}$

\begin{tabular}{ccccc}
\hline Generator & $\begin{array}{c}\text { Medium } \\
\text { between } \\
\text { the diodes }\end{array}$ & $p$, Torr & $T, \mathrm{~K}$ & $\begin{array}{c}\text { Observed onset } \\
\text { energies, meV }\end{array}$ \\
\hline $\mathrm{Al}$ & ${ }^{4} \mathrm{He}$ gas & $p_{\text {svp }}$ & 1.0 & 0.37 \\
$\mathrm{Sn}$ & ${ }^{4} \mathrm{He}$ gas & $8 \times 10^{-5}$ & 1.0 & 0.35 \\
$\mathrm{Sn}$ & ${ }^{4} \mathrm{He}$ gas & $p_{\text {svp }}$ & $1.0-1.4$ & $0.32,0.65,0.94,1.11$ \\
$\mathrm{Sn}$ & ${ }^{4} \mathrm{He}$ fluid & $p_{\text {svp }}$ & $1.2-1.4$ & $0.32,0.65$ \\
$\mathrm{Sn}$ & ${ }^{4} \mathrm{He}$ gas & $p_{\text {svp }}$ & $2.1-2.3$ & 0.38 \\
$\mathrm{Sn}(\mathrm{SiO}-$ & ${ }^{4} \mathrm{He}$ gas & $p_{\text {svp }}$ & $1.0-1.2$ & 0.32 \\
covered) & & & $1.0-1.3$ & 0.42 \\
$\mathrm{Sn}$ & ${ }^{3} \mathrm{He}$ gas & $p_{\text {svp }}$ & 1.3 & 0.39 \\
& & 1.0 & 1.26 & 0.37 \\
& & 0.3 & 1.24 & 0.39 \\
& & $8 \times 10^{-2}$ & 1.27 & 0.42 \\
\hline
\end{tabular}

${ }^{a}$ The error is $\pm 0.03 \mathrm{meV}$; in the case of the $\mathrm{Al}$ generator the error is $\pm 0.1 \mathrm{meV}, p_{\mathrm{svp}}$ is the saturated vapor pressure. 


\section{DISCUSSION}

From the experimental results we conclude that the anomalous heat transmission of a real solid-liquid He interface (i.e., the interface generator$\mathrm{He}$ or $\mathrm{SiO}-\mathrm{He}$ ) is enhanced if the energy of the incident phonons exceeds the value $E_{0} \simeq 0.32 \mathrm{meV}$. A significant contribution of direct desorption processes to the energy transfer cannot be derived from these results, mainly because the structures at 0.32 and $0.65 \mathrm{meV}$ also occur in the second-sound experiments. Further, the possibility of an abnormally high heat transmission of the metal-substrate interface must be discussed, but this can be excluded (i) with regard to the significant influence of the $\mathrm{He}$ isotope and (ii) because of phonon spectroscopic experiments in which it was observed that the phonon spectrum transmitted through isolator crystals shows characteristic minima only if generator and detector are immersed in liquid He. ${ }^{17,18}$ All these results are consistent with a Kapitza model assuming localized surface excitations, ${ }^{9}$ if we assume that the distribution function of the resonance frequencies of the hypothetical surface defects exhibits some pronounced maxima at energies $E>0.32 \mathrm{meV}$. Nevertheless, concerning the question of the microscopic nature of these defects, our experiments give no unambiguous information. Speculatively, the possibility may be considered that van der Waals attraction leads to the formation of complexes of $\mathrm{He}$ atoms and adsorbed impurities (e.g., $\mathrm{H}_{2} \mathrm{O}$, hydrocarbons). This assumption is supported by the experimentally observed isotope shift. In the case of the structure at $0.65 \mathrm{meV}$ a possible connection with the surface roton ${ }^{19,20}$ should be noted. Therefore, further work must include experiments with definitely contaminated surfaces. On the other hand, the occurrence of sharp spectral structures which may be affected by variation of the external parameters may give a new clue for the testing of theoretical models.

\section{ACKNOWLEDGMENTS}

It is a pleasure to acknowledge fruitful discussions with $\mathrm{H}$. J. Bauer, H. Kinder, D. Marx, and J. P. Toennies.

\section{REFERENCES}

1. P. L. Kapitza, J. Phys. (USSR) 4, 181 (1941).

2. I. M. Khalatnikov, Zh. Eksp. Teor. Fiz. 22, 687 (1952).

3. W. Eisenmenger and A. H. Dayem, Phys. Rev. Lett. 18, 125 (1967).

4. H. Kinder, Phys. Rev. Lett. 28, 1564 (1972).

5. H. J. Trumpp, K. Lassmann, and W. Eisenmenger, Phys. Lett. 41A, 431 (1972).

6. H. Kinder and W. Dietsche, Phys. Rev. Lett. 33, 578 (1973).

7. J. Weber, W. Sandmann, W. Dietsche, and H. Kinder, Phys. Rev. Lett. 40, 1469 (1978). 
8. H. Schubert, P. Leiderer, and H. Kinder, J. Low Temp. Phys. 39, 363 (1980).

9. H. Kinder, Physica, 107B, 549 (1981).

10. E. S. Sabisky and C. H. Anderson, Solid State Commun. 17, 1905 (1975).

11. K. Andres, R. C. Dynes, and V. Narayanamurti, Phys. Rev. A 8, 2501 (1973).

12. T. J. B. Swanenburg and J. Wolter, Phys. Rev. Lett. 31, 693 (1973).

13. M. W. Cole, Phys. Rev. Lett. 28, 1622 (1972).

14. R. J. Schweizer, M. Welte, J. Buck, and W. Eisenmenger, Verhandl. DPG 3/81, 509 (1981).

15. P. Taborek, M. Sinvani, M. Weimer, and D. Goodstein, Physica, 107B, 247 (1981).

16. M. Sinvani, P. Taborek, and D. Goodstein, Phys. Rev. Lett. 48, 1259 (1982).

17. D. Marx, Private communication (1981).

18. O. Koblinger, U. Heim, M. Welte, and W. Eisenmenger, to be published.

19. W. Thomlinson, J. A. Tarvin, and L. Passell, Phys. Rev. Lett. 44, 266 (1980).

20. H. J. Lauter, H. Wiechert, and C. Tiby, Physica, 107B, 239 (1981). 